\title{
An "aubergine" in the heart: huge native mitral valve endocarditis caused by Streptococcus agalactiae
}

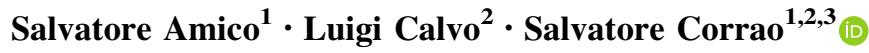

Received: 8 June 2017/ Accepted: 4 August 2017/Published online: 16 August 2017

(C) SIMI 2017

A 52-year-old woman presented to the emergency department (ED) of the ARNAS Civico, Di Cristina, Benfratelli, Palermo (Italy) in January, suffering from fluctuating fever for 3 days, up to $39^{\circ} \mathrm{C}$, associated with dyspnea/orthopnea and headache followed by vertigo and left hemiplegia. A cranial computed tomography without contrast, performed in the ED, showed focal hypodensity in the putamen of the right hemisphere; ECG showed sinus tachycardia and a posterior-anterior chest X-ray study showed mild signs of pulmonary vasculature congestion. For this reason, the patient was admitted to the internal medicine department with a diagnosis of ischemic stroke and fever.

Physical examination on admission revealed fever (temperature $38.6{ }^{\circ} \mathrm{C}$ ), tachypnea at rest (32 breaths $/ \mathrm{min}$ ), oxygen saturation of pulse oximetry measurement $83 \%$, blood pressure $120 / 65 \mathrm{mmHg}$, pulse rate 110 beats/min, no lower extremity edema or jugular venous distention. Chest auscultation revealed bilateral pulmonary crackles. Cardiac auscultation documented a grade $4 / 6$ pansystolic murmur at the mitral valve area radiated to the left anterior axillary line; for this reason, on the second day of admission,

Salvatore Corrao

s.corrao@tiscali.it

1 Department of Internal Medicine, National Relevance Hospital Trust, ARNAS Civico, Di Cristina e Benfratelli, Piazza Nicola Leotta, 4, 90127 Palermo, Italy

2 Dipartimento Biomedico di Medicina Interna e Specialistica (DiBiMIS), Centre of Research for Effectiveness and Appropriateness in Medicine (CREAM), University of Palermo, Palermo, Italy

3 Organizational, Clinical, and Traslational Research Center, Istituto Euro-Mediterraneo di Scienza e Tecnologia (I.E.ME.S.T.), Palermo, Italy bedside transthoracic echocardiogram (TTE) was performed.

The TEE revealed a giant $(4.06 \mathrm{~cm}$ maximum diameter), pedunculated, and highly mobile vegetation attached to the atrial surface of the posterior mitral leaflet, prolapsing into the left ventricle during the ventricular diastolic phase and into the left atrium during ventricular systole phase (Fig. 1).

The infection had evidently broken a chordae tendineae of the mitral subvalvular apparatus with mitral posterior leaflet flail. Consequentially, it caused a severe mitral regurgitation with an anteriorly directed eccentric jet, demonstrated by color Doppler imaging (Fig. 1e). Given the hemodynamic and embolic risk, the patient was transferred to a surgical center (IsMeTT Hospital), where the valve was removed (Fig. 1f), and a 25-mm St. Jude mitral prosthesis was implanted. The culture of the surgically resected mitral valve apparatus was positive for Streptococcus agalactiae (SA).

SA is a beta-hemolytic gram-positive bacteria. Only 2-9\% cases of Streptococcus agalactiae bacteremia present as endocarditis. The typical patient with SA endocarditis in the pre-antibiotic era was a young pregnant woman with the mitral disease; moreover, adults with significant underlying diseases with impaired immune competence, such as diabetes mellitus, alcoholism, chronic hepatic dysfunction, malignant neoplasms, AIDS, and intravenous drug use, are also prone to be infected by this microorganism [1].

SA endocarditis is characterized by acute onset, the presence of large vegetations, rapid valvular destruction, and frequent complications. The large size of the vegetations and their friability may explain the high rate of systemic emboli [2]. Moreover, it is believed that the presence of large vegetations and frequent embolic complications do 


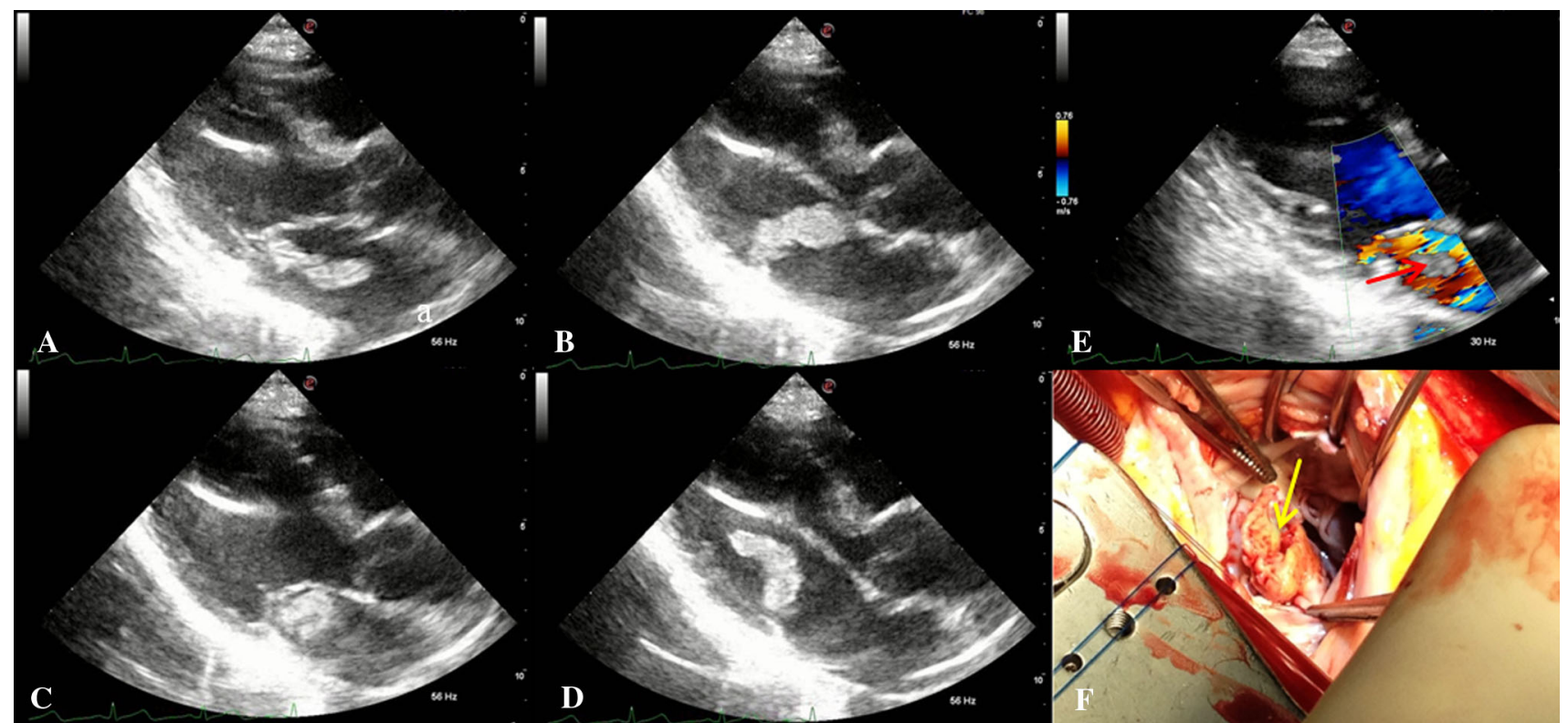

Fig. 1 Transthoracic echocardiography (parasternal long-axis view): you can see an aubergine-shaped mass (long-axis diameter $4.06 \mathrm{~cm}$ ), adhering to the mitral valve posterior leaflet, floating between the left atrium and ventricle during systole and diastole (a-d). Color Doppler

not depend on the duration of the disease, but are attributed to a lack of fibrinolysin in SA vegetations [3].

Currently, the therapy of SA endocarditis does not involve the use of steroids; furthermore, the use of thrombolytic or anticoagulant therapy in patients with embolic events in the central nervous system is controversial for increasing the risk of intracranial hemorrhage [4].

Streptococcus agalactiae is a very aggressive disease as demonstrated in the literature review, the overall mortality rate for SA infective endocarditis remains high, even despite the advent of cardiac surgery [5].

Acknowledgements SA wrote the first draft together with LC. SC supervised the case course, and corrected the first draft. SA and LC managed the case course. All the authors discussed the case and wrote the final draft.

\section{Compliance with ethical standards}

Funding This report did not receive any specific funding.

Conflict of interest All the authors have no conflict of interest.

Statement of human and animal rights All procedures performed in this case study were in accordance with the ethical standards of our institutional research committee and with the 1964 Helsinki Declaration and its later amendments or comparable ethical standards.

Informed consent Patient signed an informed consent to use clinical data for publication purposes. at the parasternal long-axis view shows severe mitral regurgitation (e) surrounding the huge vegetation mass (red arrow) and mitral valve apparatus with the giant vegetation (yellow arrow) removed during cardiac surgery (f)

\section{References}

1. Chang M, Cunha BA (2004) Streptococcus agalactiae (Group B Streptococcus) infective endocarditis complicated by myocardial abscess and heart block: case report and review. Infect Dis Clin Pract 12:107-109

2. Scully BE, Spriggs D, Neu HC (1987) Streptococcus agalactiae (group B) endocarditis: a description of twelve cases and review of the literature. Infection 15:169-176

3. Blancas D, Santin M, Olmo M, Alciade F, Carratala J, Gudiol F (2004) Group B streptococcal disease in nonpregnant adults: incidence, clinical characteristics and outcome. Eur J Clin Microbiol Infect Dis 23:168e73

4. Habib G, Lancellotti P, Antunes MJ, Bongioni MG, Casalta JP, Del Zotti F, Dulgheru R, El Khoury G, Erba PA, Iung B, Miro JM, Mulder BJ, Plonska-Gosciniak E, Price S, Roos-Hesselink J, Snygg-Martin U, Thuny F, Tornos Mas P, Vilacosta I, Zamorano JL, Reviewers Document, Erol Ç, Nihoyannopoulos P, Aboyans V, Agewall S, Athanassopoulos G, Aytekin S, Benzer W, Bueno H, Broekhuizen L, Carerj S, Cosyns B, De Backer J, De Bonis M, Dimopoulos K, Donal E, Drexel H, Flachskampf FA, Hall R, Halvorsen S, Hoen B, Kirchhof P, Lainscak M, Leite-Moreira AF, Lip GY, Mestres CA, Piepoli MF, Punjabi PP, Rapezzi C, Rosenhek R, Siebens K, Tamargo J, Walker DM (2015) 2015 ESC Guidelines for the management of infective endocarditis: The Task Force for the Management of Infective Endocarditis of the European Society of Cardiology (ESC). Endorsed by: European Association for Cardio-Thoracic Surgery (EACTS), the European Association of Nuclear Medicine (EANM). Eur Heart J 36(44):3075-3128

5. Sambola A, Miro JM, Tornos MP, Almirante B, Moreno-Torrico A, Gurgui M, Martinez E, Del Rio A, Azqueta M, Marco F, Gatell JM, The Streptococcus agalactiae Endocarditis Study Group (2002) Streptococcus agalactiae infective endocarditis: analysis of 30 cases and review of the literature, 1962-1998. Clin Infect Dis 34:1576-1584 